\title{
Elasticity, Strength and Resilience: A Comparative Study on Mechanical Signatures of $\alpha$-Helix, $\beta$-Sheet and Tropocollagen Domains
}

\author{
Markus J. Buehler $(\bowtie)$ and Sinan Keten \\ Laboratory for Atomistic and Molecular Mechanics, Department of Civil and Environmental Engineering, Massachusetts Institute of \\ Technology, 77 Massachusetts Ave. Room 1-235 A\&B, Cambridge, MA02139-4307, USA \\ Received: 29 April 2008/ Revised: 21 May 2008/Accepted: 21 May 2008 \\ (C)Tsinghua Press and Springer-Verlag 2008
}

\begin{abstract}
In biology, structural design and materials engineering is unified through formation of hierarchical features with atomic resolution, from nano to macro. Three molecular building blocks are particularly prevalent in all structural protein materials: alpha helices (AHs), beta-sheets (BSs) and tropocollagen (TC). In this article we present a comparative study of these three key building blocks by focusing on their mechanical signatures, based on results from full-atomistic simulation studies. We find that each of the basic structures is associated with a characteristic material behavior: AH protein domains provide resilience at large deformation through energy dissipation at low force levels, BS protein domains provide great strength under shear loading, and tropocollagen molecules provide large elasticity for deformation recovery. This suggests that AHs, BSs, and TC molecules have mutually exclusive mechanical signatures. We correlate each of these basic properties with the molecule's structure and the associated fundamental rupture mechanisms. Our study may enable the use of abundant protein building blocks in nanoengineered materials, and may provide critical insight into basic biological mechanisms for bio-inspired nanotechnologies. The transfer towards the design of novel nanostructures could lead to new multifunctional and mechanically active, tunable, and changeable materials.
\end{abstract}

\section{KEYWORDS}

Fracture, biology, hierarchical materials, materiomics, proteins, self-assembly, mechanical properties, theory, strength

\section{Introduction}

Protein materials are capable of unifying disparate properties such as strength (ability to sustain large stresses without fracture) and robustness (ability to undergo deformation without fracture despite the presence of defects, equivalent to toughness), as well as other dynamical properties such as self-healing ability, adaptability, changeability, and evolvability into multi-functional materials [1-4]. Many synthetic materials are not capable to unify strength and robustness, being either extremely strong with little ductility (e.g., ceramics, glass, silicon), or weak with extreme ductility (e.g., soft metals like copper) [5]. The molecular basis of these properties in protein materials remains unexplained [4], albeit it has

Address correspondence to mbuehler@MIT.EDU 
been suggested that perhaps the occurrence of these properties is due to the characteristic molecular and hierarchical features of protein materials $[1,6,7]$.

An analysis of the structure of the molecular building blocks of protein materials, facilitated by structural analysis via X-ray diffraction or nuclear magnetic resonance (NMR) spectroscopy, reveals the prevalence of three major nanostructures, or protein domains: alpha helices (AHs), beta-sheets (BSs) and tropocollagen (TC) protein domains, forming the structural basis of many protein materials [1] (the molecular structure of these three protein domains is shown in Fig. 1, left column). Table 1 provides an overview of these three basic protein structures, illustrating structural features, biological relevance and predominant occurrence of these structures. AH-based protein domains play a crucial role in the

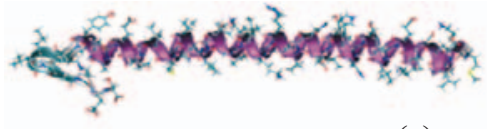

(a)
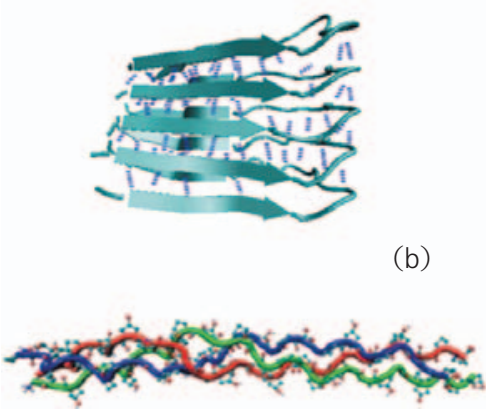

(c)

Figure 1 Overview over the three protein structures considered, with the loading conditions used to probe the mechanical signature of each structure. The AH (a) and TC molecule (c) are loaded in tensile stretch, according to the physiologically relevant loading. The BS structure (b) is loaded in shear, based on experimental analyses that have suggested the characteristic shear deformation is the most common condition (the thin grey lines in subplot (b) schematically show the $\mathrm{H}$-bond arrays in the structure)

Table 1 Overview over biological applications of the three basic protein structures AHs, BSs, and TC molecules

\begin{tabular}{|c|c|}
\hline Structure & Biological occurrence and application \\
\hline $\mathrm{AHs}$ & $\begin{array}{l}\text { Cytoskeleton (intermediate filaments-safety belt of } \\
\text { cells, actin), hair, wool, hoof }\end{array}$ \\
\hline BSs & $\begin{array}{l}\text { Muscle tissue, spider silk, fibronectin, integrins, } \\
\text { amyloids, beta-keratin in bird beaks and feather }\end{array}$ \\
\hline TC molecules & $\begin{array}{l}\text { Extracellular matrix (ECM) and therein mechanical } \\
\text { support e.g., tendon, toughening mechanisms via } \\
\text { protein phase in bone, skin, tendon }\end{array}$ \\
\hline
\end{tabular}

signaling and deformation behavior of cytoskeletal protein networks (e.g., intermediate filaments vimentin and lamin [1, 6-8]), and in determining the mechanical properties of hair, wool, hoof, and many other important structural protein materials. BS-based protein domains play a key role in spider silk [9-11], muscle tissue and are also associated with diseases, as for instance in Alzheimer's where a BS fibrous structure forms a key pathogen [12-14]. TC molecules are abundant in extracellular matrix tissue, providing elasticity and strength to tissues such as bone, tendon, and skin [3, 15-17].

While it is widely recognized that the molecular architecture of protein domains plays a decisive role in defining a protein material's properties, thus far a comparative analysis of the molecular scale mechanical properties of these most abundant protein domains has remained elusive. However, this is elementary in order to progress towards an integrated understanding of the mechanical role proteins play in biological systems, for chemomechanical coupling, signaling cascades, and protein-protein interactions [7-19], as well as to understand the behavior of protein materials based on a materials science perspective that focuses on the link between structure, property and processes (synthesis). Advancing the knowledge in this field is of significant interest for many applications in nanotechnology, since protein motifs such as AHs, BSs, and TC molecules can be used to design new materials, from bottom up [20-24].

Controlled experiments to probe the mechanics of molecules at the level of individual building blocks (molecules or protein domains), which feature ultra-small length-scale on the order of several nanometers, are difficult to carry out. Molecular simulation methods such as molecular dynamics (MD) provide a promising avenue; however, atomistic modeling is often carried out at unrealistically large deformation rates in order to observe rare events such as protein unfolding at short timescales accessible by MD [25]. Due to these computational limitations, linking the molecular structure of protein materials to key mechanical traits (elasticity, fracture, and energy dissipation) has so far remained a major challenge. 


\section{Outline of this paper}

In this paper we focus on the comparative analysis of the mechanical signatures of three basic protein structures, AHs, BSs, and TC molecules, facilitated by recently reported results of atomistic modeling of their nanomechanical response at ultra-slow deformation rates [26, 27]. These results were achieved through simulations at time-scales approaching microseconds. For AH domains, the ultra-slow dynamics of rupture under tensile load was reported in Ref. [27]. For BS domains, a similar study reported in Ref. [27] revealed the dynamical behavior under shear deformation. For TC molecules, the slow-scale deformation behavior was recently reported in Ref. [26]. All these studies led to the identification of a change in the deformation mechanism as the deformation rate is systematically reduced from ultra-fast $(>10 \mathrm{~m} / \mathrm{s})$ to $s u b-1 \mathrm{~m} / \mathrm{s}$ regimes. The most important result of these studies is the elucidation of the mechanical behavior at experimentally accessible and physiologically relevant deformation rates. However, thus far the mechanical behavior of AHs, BSs, and TC molecules has only been considered individually for each structure and has not yet been analyzed in a comparative study. This is the goal of the present paper.

\section{Results}

\subsection{Review of atomistic simulation results}

The lowest hierarchy of the three protein structures is composed of arrangements of weak H-bond interactions, organizing amino acids in the stable elementary building blocks [1, 2, 28]. The key to enable the development of an accurate bottom-up mechanistic understanding of protein domains must include an explicit description of nanopatterned $\mathrm{H}$-bond assemblies, by considering the chemical structure of the molecules. Such a description can be achieved in MD simulation models that provide a full atomistic representation of molecular structures.

All results reported in Refs. [26, 27] have been carried out with similar atomistic models, simulating the behavior of protein domains in explicit solvent at varying deformation rates. For details regarding

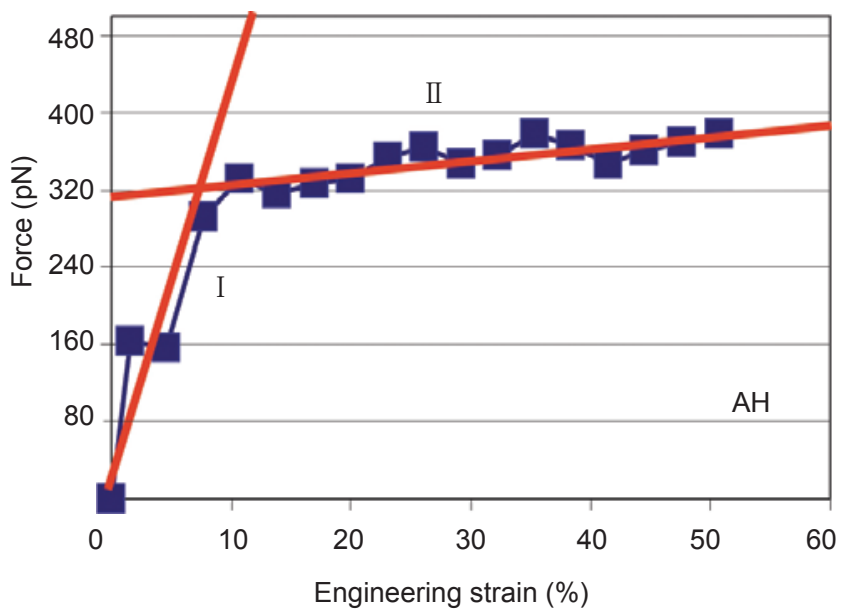

(a)

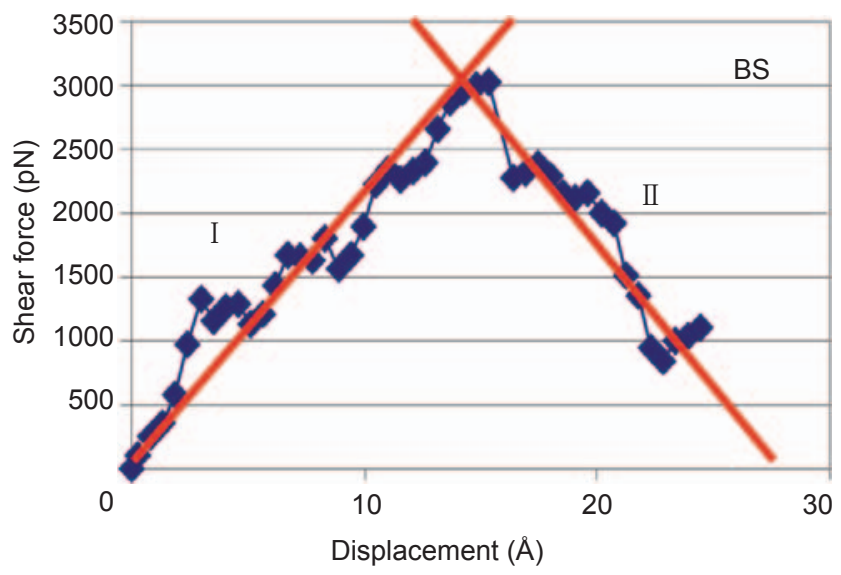

(b)

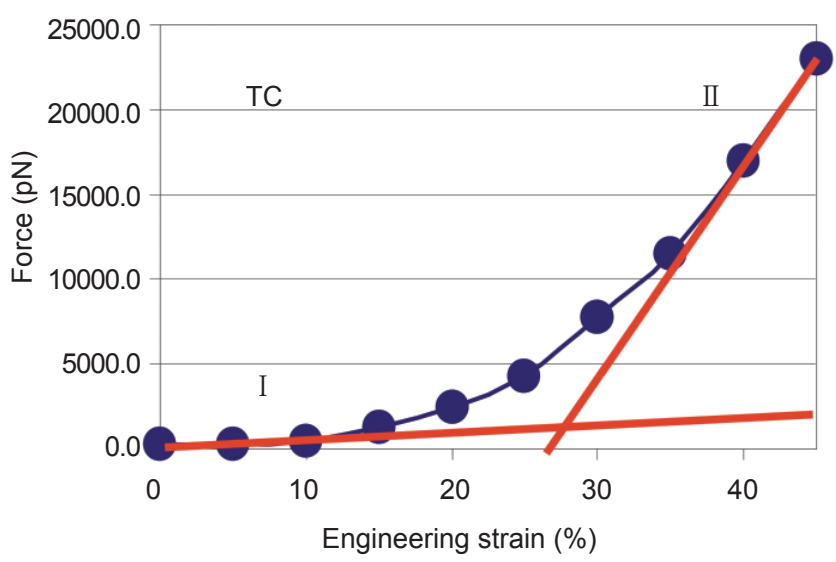

(c)

Figure 2 Comparison of the force-extension behavior of the three basic protein building blocks. Panel (a) shows the results for the $\mathrm{AH}$ structure, panel (b) the results for the BS structure, and panel (c) the results for the TC molecule. The loading conditions are as shown in Fig. 1, right column. The red continuous lines show a bilinear fit to the small-strain and large-strain elastic properties, marking the smalldeformation (I) and large-deformation (II) regimes 
the atomistic model and computational approach, we refer the reader to the original publications [26, 27] (here we provide only a brief review of the most important aspects).

The mechanical loading conditions of the three protein structures are shown in Fig. 1, right column. As illustrated in this figure, the AH structure (Fig. 1(a)) and the TC structure (Fig. 1(c)) are both loaded in tension. This loading condition is motivated based on the physiologically relevant loading condition due to mechanical stimuli of larger-scale tissue features (see, e.g., Ref. [29]). The BS structure (Fig. 1(b)) is loaded in shear, also following the physiologically and experimentally relevant loading condition.

The characteristic force-extension behavior curves are shown in Fig. 2, for the AH, BS, and TC structures. This figure shows representative force-extension histories at ultra-slow deformation rates (the $\mathrm{AH}$ structure is loaded at $0.1 \mathrm{~m} / \mathrm{s}$, the BS structure at $0.05 \mathrm{~m} / \mathrm{s}$, and the TC structure at $0.1 \mathrm{~m} / \mathrm{s}$ ). We do not aim to provide a quantitative analysis pertaining to a specific deformation rate, and therefore we do not discuss the forceextension histories at different rates. Rather, here we aim at a qualitative analysis of the distinct features of each structure's mechanical signature. Even though the rupture force depends on the rate for the $\mathrm{AH}$ and BS structure, the overall shape of the curves shown in Figs. 2(a) and (b) is independent of the deformation speed [27] at low pulling rates. Further, for the TC molecule it is found that for deformation speeds $<1 \mathrm{~m} / \mathrm{s}$ the force-extension behavior does not depend on loading rate [26], leading to a convergence of the curves to the result shown in Fig. 2(c).

\subsection{Comparative analysis}

In the case of the $\mathrm{AH}$ structure, an initial linear elastic regime is followed by a large plateau regime at which the helix "slowly" uncoils, releasing one turn at an time, as shown in Fig. 3(a). The analysis of MD simulations has shown that approximately three $\mathrm{H}$-bonds in a turn break simultaneously [27]. The long linear array of turns in an $\mathrm{AH}$ protein domain (each stabilized by approximately $3 \mathrm{H}$-bonds) provides for extremely large plastic displacements and therefore strains that reach $125 \%$ to $150 \%$ during the plateau regime. This plateau regime is characterized by an almost flat force-extension behavior and very low tangent slope. The forces in the plateau regime are quite small, not exceeding a few hundreds $\mathrm{pN}$ and less. This behavior is similar to those observed in experimental studies $[30,31]$. It is noted that the plateau regime is followed by a steep increase in the force-extension relation once the backbone covalent bonds are being stretched, upon complete uncoiling of the $\mathrm{AH}$ structure [32, 33] (this behavior is not explicitly shown in Fig. 3(a) as we

\begin{tabular}{c|c|c|c|c}
\hline & \multicolumn{4}{|c}{ Mechanical trait (signature) } \\
\hline Structure & $\begin{array}{l}\text { Elasticity (ability } \\
\text { to store and reuse } \\
\text { energy) }\end{array}$ & $\begin{array}{l}\text { Energy dissipation } \\
\text { (ability to dissipate } \\
\text { elastic energy) }\end{array}$ & $\begin{array}{l}\text { Tolerate large } \\
\text { displacement }\end{array}$ & $\begin{array}{l}\text { Fracture strength } \\
\text { (H-bond rupture) }\end{array}$ \\
\hline AHs & 0 & 3 & 3 & 0 \\
\hline BSs & 1 & 1 & 0 & 3 \\
\hline TC & 3 & 1 & 1 & 1 \\
\hline
\end{tabular}

(a)

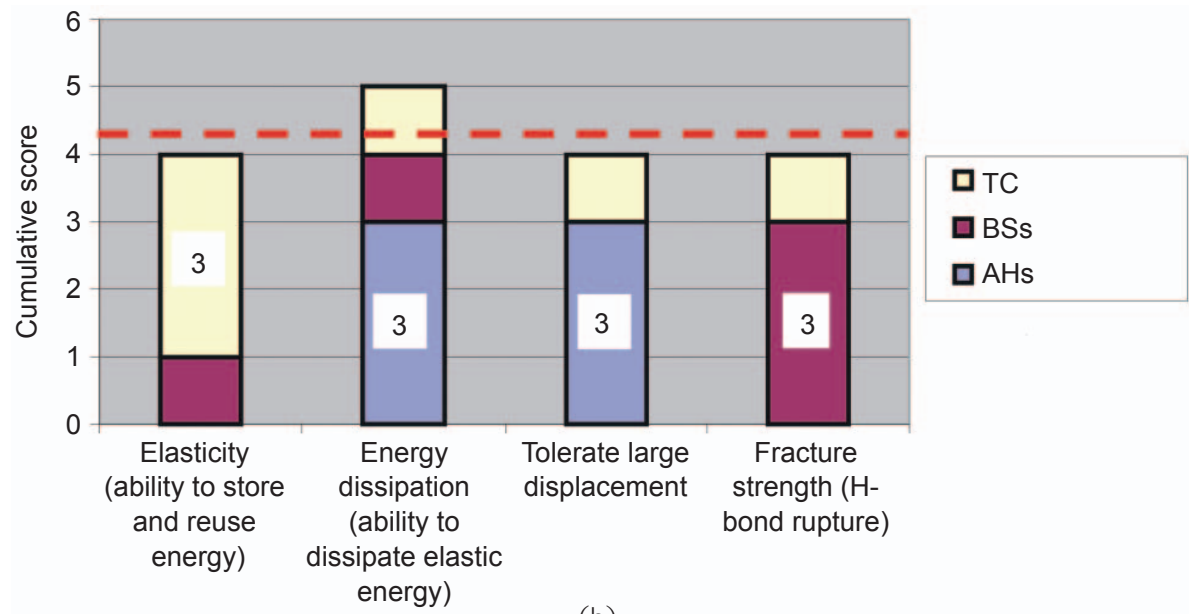

(b)

Figure 3 Summary of key mechanical traits of the three basic protein structures (qualitatively measured in: $3=$ large, $2=$ medium, $1=$ small, $0=$ little/no). Subplot (a) shows a table with associated values listed, and subplot (b) depicts a graph plotting the specific traits for all there structures. The cumulative score of each feature is shown in subplot (b). The analysis shows that the cumulative score remains approximately constant, with an average value of 4.25 , for all properties. The graph indicates that the three structures have mutually exclusive properties 
focus on the regime dominated by H-bond rupture). Rupture of the $\mathrm{AH}$ structure after stretching the covalent backbone occurs at force levels of 5-7 $\mathrm{nN}$ [32].

The rupture behavior of the BS structure shows a quite different behavior, featuring a characteristic peak that is reached at approximately $15 \AA$ shear deformation (corresponding to approximately 35\% shear strain). The graph shows a linear-elastic increase followed by a peak and a negative slope in the forceextension plot, marking the onset of the mechanical instability. This behavior is reminiscent of a brittle structure; the force peaks are rather large (exceeding several $\mathrm{nN}$, even at extrapolations to much slower deformation rates [27]), followed by a sudden drop in force. The unit molecular mechanism of fracture is the rupture of $\mathrm{H}$-bonds that bind stacked beta-sheet strands in clusters of 3-4. Once the clusters of H-bonds have broken, the structure fails catastrophically and the force drops to zero rather quickly.

The TC molecule shows yet another characteristic mechanical signature. The TC force-extension plot shows continuous stiffening. Starting from a very small tangent modulus (vanishing at zero deformation when the molecule is in a slightly crimped configuration), the modulus increases continuously to $4 \mathrm{GPa}$ at approximately $8 \%$ strain, to $40-60 \mathrm{GPa}$ at large deformation in the $30 \%-50 \%$ strain regime [26]. Forces reached in this regime approach several tens of $\mathrm{nN}$. Rupture of the covalent backbone, which dominates the elasticity at the stiffest regime, reaches approximately $20 \mathrm{nN}$ [34, 35] (this is a higher value than in the case of a single $\mathrm{AH}$, due to the parallel loading of three polypeptide chains; this behavior is not shown in Fig. 2(c)). Rupture of H-bonds dominates the deformation mechanism in the regime of $10 \%-30 \%$ strain (it begins when the molecule is straightened out, at $10 \%$ strain [26], and ends when approximately $30 \%$ strain is reached, when all $\mathrm{H}$-bonds are broken). The breaking of $\mathrm{H}$-bonds is coupled to a rotation of the ends of the molecule to accommodate the uncoiling mechanisms of the triple helix. The characteristic triple helical structure provides the structural basis for this continuous stiffening behavior. Unlike the $\mathrm{AH}$ in which the stretching deformation is accommodated by rupture of turns, the triple helix provides self-reinforcing character. In the case of AHs, polypeptide length is "freed" upon rupture of the cluster of H-bonds. This does not happen in the case of TC, where uncoiling must occur along with breaking of $\mathrm{H}$-bonds, leading to a continuous increase in stiffness.

This comparative description of the nanomechanics of AHs, BSs and TC molecules reveals that all three structures have in common:

(1) The initial structure of the molecule is lost under heavy deformation,

(2) Deformation is accommodated by rupture of H-bonds, representing the elementary mechanism of deformation (similar to dislocations in metals),

(3) The behavior is strongly nonlinear elastic, that is, hyperelastic (hyperelasticity refers to the phenomenon of change in tangent stiffness as a function of deformation).

However, there are major differences in the mechanical signature of each of these structures, most notably in

(1) Type of the hyperelastic behavior (stiffening versus softening),

(2) Force levels at the rupture point,

(3) Maximum plastic (H-bond mediated) strains (displacements),

(4) Nature of instability at breaking point (e.g. followed by negative slope or continuous stiffening).

Table 2 provides a quantitative analysis of the behavior discussed in the previous three paragraphs, focusing on the modulus at small and large deformation, the maximum plastic strain, and the characteristics of the hyperelastic features. The analysis summarizes the key differences between the three protein structures considered here. $\mathrm{AH}$ protein domains are capable of tolerating the largest deformation without fracturing, at relatively small force levels. BS domains provide a significantly higher mechanical resistance (approximately 10 times larger than the maximum force measured in AHs), but BS structures are not capable of tolerating large deformation. BSs break in a more brittle fashion, leading to a sudden drop in force once the maximum displacement is reached. TC molecules show a characteristic continuous stiffening behavior as deformation increases. The progressive stiffening and relatively large force levels provide significant elastic 
Table 2 Quantitative comparison of mechanical properties of AHs, BSs, and TC molecules, including maximum plastic strain, hyperelastic characteristics, as well as small-strain and large-strain elastic moduli. The hyperelastic characteristics refers to the type of the nonlinear stressstrain behavior, that is, whether the material softens or stiffens with strain and what sequence of stiffness change occurs during large deformation

\begin{tabular}{|c|c|c|c|c|}
\hline Structure & $\begin{array}{l}\text { Maximum plastic strain } \\
\text { (all H-bonds broken) }\end{array}$ & Hyperelastic characteristic & Small-strain elastic modulus ${ }^{\mathrm{a}}$ & $\begin{array}{l}\text { Large-strain elastic } \\
\text { modulus }^{\mathrm{a}}\end{array}$ \\
\hline AHs & $125 \%-150 \%$ & $\begin{array}{l}\text { Discrete softening (stiffening at very large } \\
\text { deformation }>200 \% \text { ) }\end{array}$ & $2.5 \mathrm{GPa}$ & $0.1 \mathrm{GPa}$ \\
\hline BSs & $\begin{array}{l}\text { 35\% (15 A shear } \\
\text { displacement) }\end{array}$ & Softening (instability at rupture point) & $240 \mathrm{pN} / \AA ̊$ & Negative slope \\
\hline TC & $30 \%$ & Stiffening (continuous) & $0.4 \mathrm{GPa}$ (4 GPa at 8\% strain) & $20.40 \mathrm{GPa}$ \\
\hline
\end{tabular}

${ }^{a}$ the modulus for $\mathrm{AH}$ and TC structures is obtained by normalization of the force by the cross-sectional area of the protein domain, for details we refer the reader to the literature $[26,27]$

energy storage and recovery. No sudden, catastrophic failure is observed.

Figure 3 shows a summary of key mechanical traits of the three basic protein structures. The traits considered here are: elasticity (that is, the ability to store and reuse mechanical energy), energy dissipation (ability to dissipate elastic energy), tolerating large displacements (that is, the ability to tolerate large displacements), and fracture strength due to rupture of H-bond clusters (that is, the maximum strength at the point when all $\mathrm{H}$-bond clusters are broken). The comparison of the three structures is achieved by assigning a score to each property, measuring how much of the mechanical trait is realized by the particular protein structure. The applicability is qualitatively measured in: $3=$ large, $2=$ medium, $1=$ small, and $0=$ little/no. Figure. $3(\mathrm{a})$ shows a table with associated values listed, and subplot (b) depicts a graph plotting the specific traits for all there structures. The analysis shows that the occurrence of the highest rating " 3 " can be uniquely assigned to specific structures: elasticity is associated with $\mathrm{TC}$, energy dissipation and large displacements (the combination=resilience) are associated with $\mathrm{AH}$, and fracture strength (H-bonds) is associated with BS. The cumulative score of each feature is shown in Fig. 3 , also marking the contributions of each structure. The analysis shows that the cumulative score remains approximately constant, $4.5 \pm 0.5$, for all properties. The graph suggests that the three structures have mutually exclusive mechanical signatures.

\section{Summary}

Our analysis suggests that the three basic protein structures can each be associated with key characteristic mechanical traits: AHs are resilient structures that tolerate large displacements (strains up to $150 \%$ and more before fracture of the protein backbone occurs), and are capable of dissipating large energy, in particular in cyclic loading when a hysteresis behavior is observed (for details, please see [33]). BSs are characterized by strength, providing great resistance when loaded in shear, albeit at relatively small displacements. TC molecules are highly elastic stiffening structures that can be stretched up to $50 \%$ strain and can store quite large amounts of elastic energies before fracture of the protein backbone occurs.

This characterization enables us to assign a single most important mechanical trait to each structure: AHs are characterized by resilience, BSs by strength, and TC molecules by elasticity (elastic deformation recovery). Figure 4 shows a "triangle" of these properties in a graphical representation. This classification, based on the systematic comparative analysis shown in Fig. 2 and the graphical analysis shown in Fig. 3, is the most important contribution of this paper. The observation that the three most prevalent basic protein structures have mutually exclusive mechanical signatures may explain their biological significance. These three protein topologies form unique structural elements with characteristic mechanical roles similar to typical load bearing members in building design such as cables, trusses, and concrete shear walls. Multi-functional materials are created by combination of the basic structures into composite materials, which enables one to create structures that combine features associated with each structural element in a single material. This may 


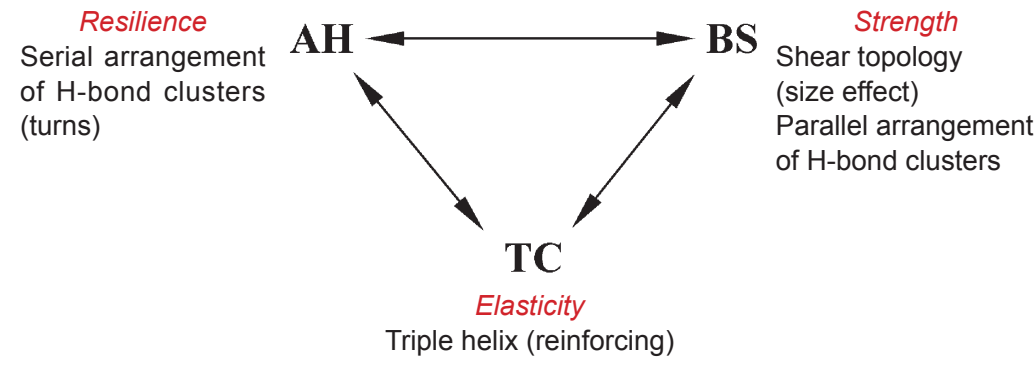

Figure 4 Resilience-strength-elasticity triangle, summarizing the key mechanical features of the three basic building blocks and their key structural feature that is associated with these features

explain the observation of multi-functionality and the combination of seemingly incompatible properties (such as strength and robustness) in biological protein materials. The transfer of this insight to the design of novel nanostructures could lead to new multifunctional and mechanically active, tunable and changeable materials.

\section{Discussion and conclusions}

The analysis reported here, focusing on strength, elasticity, and hyperelastic features, is, to the best of our knowledge, the first analysis of its kind and sheds light onto the most basic features at the level of single molecules. The comparison of the three structures was facilitated by the availability of a set of simulation results that were obtained using a consistent set of atomistic models.

The results are significant for the development of the mechanome [19] of the three basic protein structures. The advancement of this field is crucial for studies of biological systems, disease diagnosis, and treatment, as well as the design of novel biomaterials. It is key to reverse-engineer the human body, the key to understand diseases at multi-scale levels (cancer, genetic diseases, infectious diseases), to enable advanced treatments (intervention at level of relevant proteins, nanomedicine, protein hierarchies, biomaterials) and diagnostics (mechanical disease signature, protein misregulation).

Figure. 5 shows an overview over examples of larger-scale assembly structures of the three basic protein building blocks considered here, alpha helices, beta-sheets and tropocollagen molecules. For each case, a specific biological protein material is selected. Alpha helices form cellular protein filaments such as intermediate filaments (Fig. 5(a)). Beta-sheets are commonly observed in spider silk fibers (Fig. 5(b)). Tropocollagen molecules assemble into fibrils and fibers in collagenous tissues such as tendon (Fig. 5(c)). Each of the three structures has characteristic material properties. Intermediate filament networks are mechanically resilient structures that provide strength to the cell under large deformation. Spider silk is a very strong yet very elastic material due to the presence of beta-sheet nanocrystals dispersed in a semi-ordered biopolymer matrix. Collagenous connective tissue such as tendon or bone are highly elastic yet tough structural materials. The multi-scale arrangement of constituents in the three examples plays a key role in determining the mechanical properties at larger length-scales, and it must be explicitly taken into consideration in order to understand the unique design of these biological materials. Such analyses are left to future studies.

After identifying the entire genetic code of several species, a grand challenge remains the understanding of the multiscale behavior of hierarchical protein assemblies, and applying the generated knowledge to the design of novel synthetic materials. The elucidation of the interface between structure and material provides the need for a systematic and rigorous materials science-based analysis of protein materials, an effort we have defined as "materiomics". Materiomics is defined as the study of the material properties of hierarchical protein structures and their effect on the macroscopic function, elucidating how structural features across the scales contribute in defining a material's properties.

Through these efforts, the understanding the fundamental design laws of hierarchical protein materials enables us to link the structural protein organization to the appropriate biological functions. The transfer of this insight to other technological materials could allow an extended use of hierarchies in bioinspired or biomimetic synthetic materials at nanoscale, such as hierarchically organized CNTbundles, nanowires or polymer-protein composite 


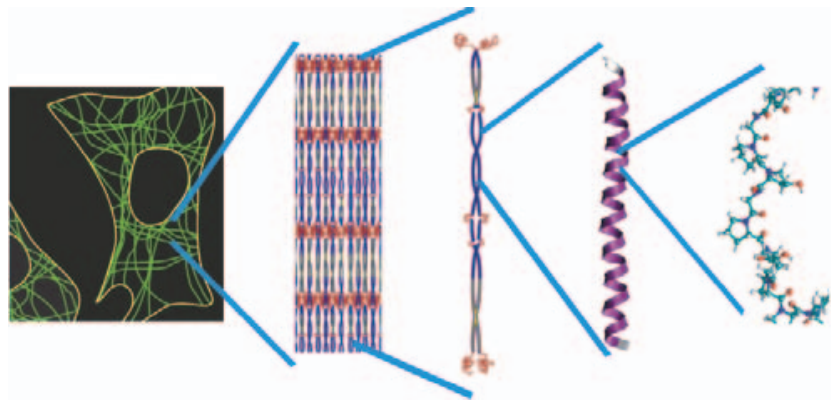

(a)

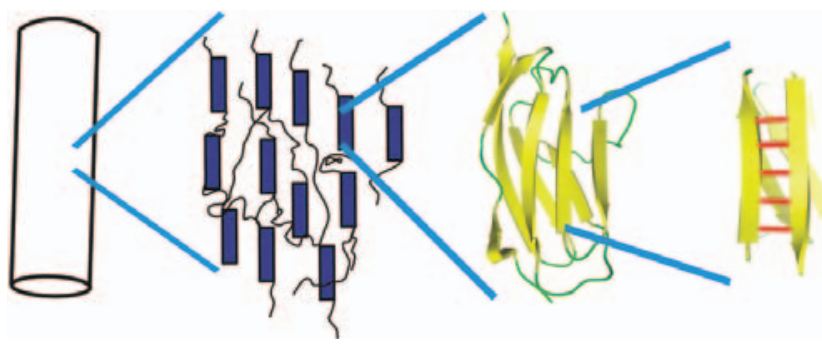

(b)

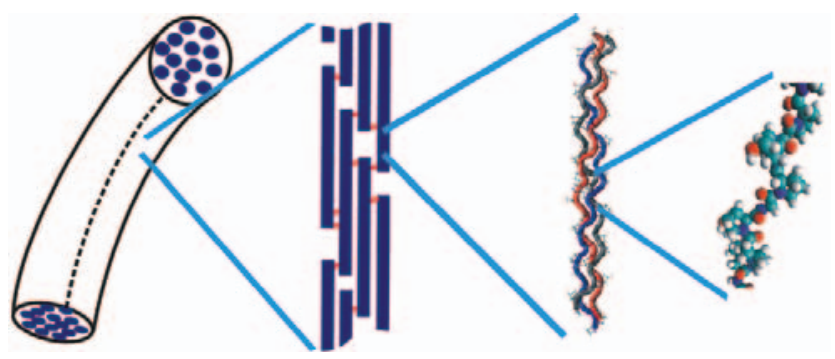

(c)

Figure 5 Overview over examples of larger-scale assembly structures of the three basic protein building blocks considered here, alpha-helices (a), beta-sheets (b) and tropocollagen molecules (c). Alpha-helices form cellular protein filaments such as intermediate filaments. Betasheets assemble into nanocrystalline composite structures in spider silk. Tropocollagen molecules assembly into fibrils and fibers in collagenous tissues such as tendon. Each of the three structures has characteristic properties. Intermediate filament networks are mechanically resilient structures that provide strength to the cell under large deformation. Spider silk is a very strong yet very elastic material. Collagenous tissues such as tendon or bone are highly elastic yet tough structural materials. The multi-scale arrangement of constituents in the three examples plays a key role in determining the mechanical properties at larger length-scales, and it must be explicitly taken into consideration in order to provide an understanding of each specific tissue type

$[36,37]$. This is vital for the development and application of new organic and organic-inorganic composites, which will mainly consist of elements that appear in our environment in virtually unlimited amount $(\mathrm{C}, \mathrm{H}, \mathrm{N}, \mathrm{O}, \mathrm{S})$. Recent reports provide evidence that it is possible to synthesize bundles of AH-based coiled-coil proteins in a controlled manner
[20], and many other peptide synthesis techniques are progressing rapidly [20-24]. Our model combined with these new manufacturing techniques may be the first step towards a de novo bottom-up structural design for new nanotechnology applications.

\section{Acknowledgements}

This research was supported by the Army Research Office, grant number W911NF-06-1-0291 (program officer Dr. Bruce LaMattina) and by a National Science Foundation CAREER Award (program manager Dr. Jimmy Hsia). Sinan Keten acknowledges the Presidential Fellowship Program and additional support from the Department of Civil and Environmental Engineering at Massachusetts Institute of Technology (MIT).

\section{References}

[1] Alberts, B.; Bray, D.; Lewis, J.; Raff, M.; Robert, K.; Watson, J. D. Molecular Biology of the Cell; Taylor \& Francis: New York, NY, 2002.

[2] Buehler, M. J.; Keten, S.; Ackbarow, T. Theoretical and computational hierarchical nanomechanics of protein materials: Deformation and fracture. Prog. Mater. Sci., in press.

[3] Fratzl, P.; Weinkamer, R. Nature's hierarchical materials. Prog. Mater. Sci. 2007, 52: 1263-1334.

[4] Smith, B. L.; Schäffer, T. E.; Viani, M.; Thompson, J. B.; Frederick, N. A.; Kindt, J.; Belcher, A.; Stucky, G. D.; Morse, D. E.; Hansma, P. K. Molecular mechanistic origin of the toughness of natural adhesives, fibres and composites. Nature 1999, 399(6738): 761-763.

[5] Hirth, J. P.; Lothe, J. Theory of Dislocations; WileyInterscience: New York, NY, 1982.

[6] Herrmann, H.; Aebi, U. Intermediate filaments: Molecular structure, assembly mechanism, and integration into functionally distinct intracellular scaffolds. Ann. Rev. Biochem. 2004, 73: 749-789.

[7] Rowat, A. C. Lammerding, J.; Herrmann, H.; Aebi, U. Towards an integrated understanding of the structure and mechanics of the cell nucleus. Bioessays 2008, 30(3): 226-36.

[8] Kreplak, L.; Fudge, D. Biomechanical properties of intermediate filaments: From tissues to single filaments 
and back. Bioessays 2007, 29(1): 26-35.

[9] Du, N.; Liu, X. Y.; Narayanan, J.; Li, L.; Lim, M. L. M.; Li, D. Q. Design of superior spider silk: From nanostructure to mechanical properties. Biophys. J. 2006, 91(12): 45284535.

[10] Vollrath, F.; Porter, D. Spider silk as archetypal protein elastomer. Soft Matter. 2006, 2(5): 377-385.

[11] Termonia, Y. Molecular modeling of spider silk elasticity. Macromolecules 1994, 27(25): 7378-7381.

[12] Mostaert, A. S.; Higgins, M. J. Nanoscale mechanical characterisation of amyloid fibrils discovered in a natural adhesive. J. Biol. Phys. 2006, 32(5): 393-401.

[13] Smith, J. F.; Knowles, T. P. J.; Dobson, C. M.; MacPhee, C. E.; Welland, M. E. Characterization of the nanoscale properties of individual amyloid fibrils. P. Natl. Acad. Sci. U. S. A. 2006, 103(43): 15806-15811.

[14] Slotta, U.; Hess, S.; Spiess, k.; Stromer, T.; Serpell, L.; Scheibel, T. Spider silk and amyloid fibrils: A structural comparison. Macromol. Biosci. 2007, 7(2):183-188.

[15] Ricard-Blum, S.; Ruggiero, F.; van der Rest, M. The collagen superfarmily. Collagen. 2005, 35-84.

[16] Gelse, K.; Poschl, E.; Aigner; T. Collagens — structure, function, and biosynthesis. Adv. Drug Delivery Rev. 2003, 55(12): 1531-1546.

[17] Vincent, J. F. V. Structural Biomaterials; Princeton University Press: Princeton, NJ, 1990.

[18] Ingber, D. E. Cellular mechanotransduction: Putting all the pieces together again. FASEB J. 2006, 20(7): 811-827.

[19] Lang, M. In Frontiers of Engineering: Reports on LeadingEdge Engineering from the 2007 Symposium; National Academy of Engineering of the National Academies, 2008.

[20] Papapostolou, D.; Smith, A. M.; Atkins, D. T.; Oliver, S. J.; Ryadnov, M. G.; Serpell, L. C.; Woolfson, D. N. Engineering nanoscale order into a designed protein fiber. P. Natl. Acad. Sci. USA 2007, 104(26): 10853-10858.

[21] Langer, R.; Tirrell, D. A. Designing materials for biology and medicine. Nature 2004, 428(6982): 487-492.

[22] Smeenk, J. M.; Otten, M. B.; Thies, J.; Tirrell, D. A.; Stunnenberg H. G.; van Hest J. C. Controlled assembly of macromolecular beta-sheet fibrils. Angew. Chem. Int. Ed. Engl. 2005, 44(13): 1968-1971.

[23] Zhao, X. J.; Zhang, S. G. Molecular designer selfassembling peptides. Chem. Soc. Rev. 2006, 35(11): 1105-1110.

[24] Mershin, A.; Cook, B.; Kaiser, L.; Zhang, S. G. A classic assembly of nanobiomaterials. Nat. Biotechnol. 2005,
23(11): 1379-1380.

[25] Sotomayor, M.; Schulten, K. Single-molecule expzeriments in vitro and in silico. Science 2007, 316(5828): 1144-1148.

[26] Gautieri, A.; Buehler, M. J.; Redaelli, A. Deformation rate controls elasticity and unfolding pathway of single tropocollagen molecules. J. Mechanical Behavior of Biomedical Materials, in press. [online early access]. DOI: 10.1016/j.jmbbm.2008.03.001.

[27] Ackbarow, T.; Chen, X. F.; Keten, S.; Buehler, M. J. Hierarchies, multiple energy barriers and robustness govern the fracture mechanics of alpha helical and betasheet protein domains. P. Natl. Acad. Sci. USA 2007, 104: 16410-16415

[28] Ackbarow, T.; Buehler, M. J. Nanopatterned protein domains unify strength and robustness through hierarchical structures, in submission.

[29] Buehler, M. J. Nature designs tough collagen: Explaining the nanostructure of collagen fibrils. P. Natl. Acad. Sci. USA 2006, 103(33): 12285-12290.

[30] Schwaiger, I.; Sattler, C.; Hostetter, D. R.; Rief, M. The myosin coiled-coil is a truly elastic protein structure. Nat. Mater. 2002, 1(4): 232-235.

[31] Root, D. D.; Yadavalli, V. K.; Forbes, J. G.; Wang, K. Coiled-coil nanomechanics and uncoiling and unfolding of the superhelix and alpha helices of myosin. Biophys. J. 2006, 90(8): 2852-2866.

[32] Buehler, M. J. Hierarchical chemo-nanomechanics of stretching protein molecules: Entropic elasticity, protein unfolding and molecular fracture. J. Mech. Mater. Struct. 2007, 2(6): 1019-1057.

[33] Ackbarow, T.; Buehler, M. J. Superelasticity, energy dissipation and strain hardening of vimentin coiled-coil intermediate filaments: Atomistic and continuum studies. J. Mater. Sci. 2007, 42(21): 8771-8787.

[34] Buehler, M. J.; Wong, S. Y. Entropic elasticity controls nanomechanics of single tropocollagen molecules. Biophys. J. 2007, 93(1): 37-43.

[35] Buehler, M. J. Atomistic and continuum modeling of mechanical properties of collagen: Elasticity, fracture and self-assembly. J. Mater. Res. 2006, 21(8): 1947-1961.

[36] Cui, X. Q.; Li, C. M.; Zang, J. F.; Zhou, Q.; Gan, Y.; Bao, H. F.; Guo, J.; Biocatalytic generation of ppy-enzyme-CNT nanocomposite: From network assembly to film growth. J. Phys. Chem. C 2007, 111(5): 2025-2031.

[37] Winey, K. I.; Vaia R. A. Polymer nanocomposites. MRS Bulletin 2007, 32(4): 5.

\section{国显 Springer}

